
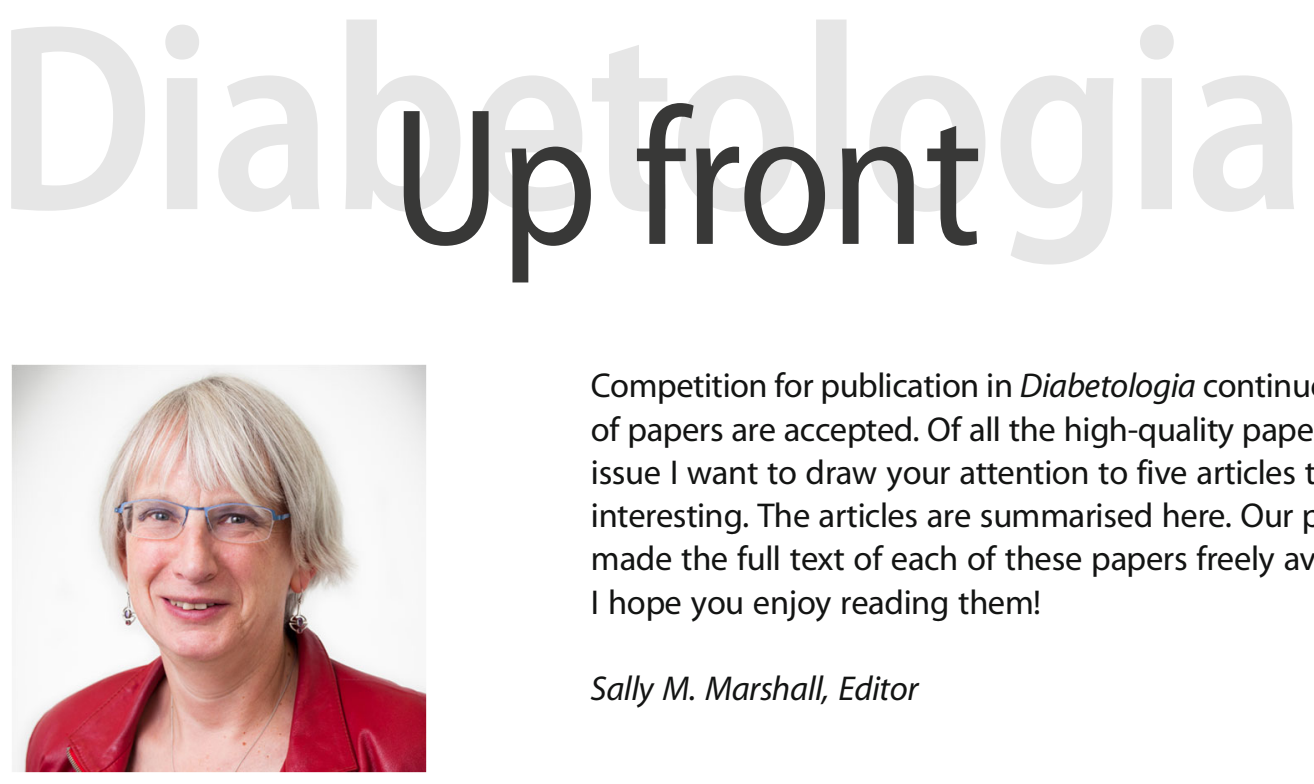

Competition for publication in Diabetologia continues to grow, and less than 20\% of papers are accepted. Of all the high-quality papers that appear in this month's issue I want to draw your attention to five articles that I think are particularly interesting. The articles are summarised here. Our publisher, Springer, has kindly made the full text of each of these papers freely available.

I hope you enjoy reading them!

Sally M. Marshall, Editor

\section{Diabetes at the crossroads: relevance of disease classification to pathophysiology and treatment}

\section{R. David Leslie, Jerry Palmer, Nanette C. Schloot, Ake Lernmark}

Every good clinician can distinguish type 1 (insulindependent) diabetes from type 2 (non-insulin dependent) diabetes, or at least they think they can. But the Boolean logic that underpins this traditional classification has recently been confounded by advances in genetics, immunology and biochemistry. Widely available laboratory tests have revealed the kaleidoscopic nature of diabetes, despite clinical phenotypes that are apparently straightforward. Allied to different causes are different outcomes and the need for distinct therapy. Such personalised treatment targets invoke personalised treatment strategies to be accommodated within differential responses to pharmaceuticals. In this issue, Leslie and colleagues provide a review of recent advances in laboratory medicine in the context of clinical diabetes. As with other diseases, diabetes stands at a crossroads where the future points towards an era of precision medicine with a better understanding and better treatment of this disease in its myriad forms.
CTLA4-Ig in B7-1-positive diabetic and non-diabetic kidney disease

Roberto Bassi, Alessia Fornoni, Alessandro Doria, Paolo Fiorina

The B7 pathway, a critical regulator of T cell-mediated immune function, has been identified in podocytes, potentially implicating this pathway in the onset of proteinuric kidney disease. Pharmacological targeting of B7 receptors on podocytes with the fusion protein CTLA4-Ig actively modulates podocyte structure and function in vitro and in animal models, and thus influences proteinuria. In a review in this issue, Bassi et al summarise recent advances in the understanding of the immune-relevant abilities of podocytes in the context of B7-1-positive diabetic and non-diabetic kidney disease and the clinical applications for CTLA4-Ig in kidney pathology, with a particular focus on diabetic kidney disease. The authors also envision the application of CTLA4-Ig therapy in a subset of B7-1-positive diabetic patients with mild kidney dysfunction. Finally, recent reports of inconsistent podocyte B7-1 detection and non-linear efficacy of CTLA4-Ig in proteinuric patients are discussed, highlighting the urgency of establishing a consensus for protocols for B7-1 detection and CTLA4-Ig therapy. 
Type 2 diabetes and leucocyte DNA methylation: an epigenome-wide association study in over 1,500 older adults

Ines Florath, Katja Butterbach, Jonathan Heiss, Melanie Bewerunge-Hudler, Yan Zhang, Ben Schöttker, Hermann Brenner

DNA methylation has been associated with stable alterations in gene expression. In this issue, Florath et al report on a study in which DNA methylation was measured in whole blood samples from 1,515 participants from a population-based cohort of elderly German adults, 240 of whom had type 2 diabetes. Using the Illumina Infinium HumanMethylation450 BeadChip, an association between methylation at the CpG site cg19693031 and type 2 diabetes was identified and replicated in an independent subcohort. Decreasing cg19693031 methylation was observed with increasing fasting glucose concentrations and $\mathrm{HbA}_{1 \mathrm{c}}$. Cg19693031 is located within the 3'-untranslated region of the gene encoding thioredoxin-interacting protein (TXNIP). Given that TXNIP levels are increased in hyperglycaemic animals and human adipose tissue incubated with glucose, cg19693031 might play a role in the pathophysiology of type 2 diabetes by altering the expression of TXNIP.

High intensity intermittent exercise improves cardiac structure and function and reduces liver fat in patients with type 2 diabetes: a randomised controlled trial

Sophie Cassidy, Christian Thoma, Kate Hallsworth, Jehill Parikh, Kieren G. Hollingsworth, Roy Taylor, Djordje G. Jakovljevic, Michael I. Trenell

Heart disease is the leading cause of mortality in type 2 diabetes. Early changes in left ventricular structure and function have been identified prior to any overt heart disease, yet therapies to target cardiac dysfunction in this patient group are sparse. In this issue, Cassidy et al present the results of their study of 12 weeks of a high intensity intermittent exercise training (HIIT) intervention in patients with type 2 diabetes. Participants in the HIIT group $(n=12)$ showed significant improvements in systolic and diastolic function, increases in left ventricular wall mass and enddiastolic blood volume, and reductions in cardiac torsion compared with those in the standard therapy group $(n=11)$. The reductions in liver fat in the HIIT group are the largest to be demonstrated following an exercise intervention to date and suggest that this type of exercise targets fat depots that play a role in type 2 diabetes aetiology. These findings are the first to indicate that HIIT holds potential as a clinical therapy to moderate cardiac risk and reduce liver fat in adults with type 2 diabetes.

Role of microRNAs in the age-associated decline of pancreatic beta cell function in rat islets

Ksenia Tugay, Claudiane Guay, Ana C. Marques, Florent Allagnat, Jonathan M. Locke, Lorna W. Harries, Guy A. Rutter, Romano Regazzi

Ageing is associated with a progressive decline in the secretory activity of beta cells and with a reduction in the capacity of these cells to expand in response to insulin resistance. Age-associated beta cell dysfunction is believed to be linked to changes in gene expression. In this issue, Tugay et al report the results of a study that compared the microRNA (miRNA) expression profiles of pancreatic islets isolated from young and old rats. The levels of several miRNAs were different between the two groups. Transfection of the islets of young animals with miRNA mimics or anti-miRNAs to mirror the changes with ageing in specific microRNAs produced the same alterations in gene expression as observed in the older animals, resulting in impaired beta cell proliferation. In view of these findings, the authors suggest that alterations in miRNA levels that occur during ageing could contribute to the inability of beta cells to expand under conditions of increased insulin demand, favouring the development of type 2 diabetes.

All text supplied by the authors. 